\title{
THE REMOTE SCIENTIST
}

\author{
J. Samson, L. Peterson, L. Harmon, D. Conrad \\ NASA Space Applications Technology Center \\ ERIM \\ P.O. Box 134001 \\ Ann Arbor, MI 48113-4001 \\ 313-994-1200
}

\author{
K. Clark \\ Department of Anatomy and Cell Biology \\ 5805 Medical Science II \\ The University of Michigan Medical School \\ Ann Arbor, MI 48109-0616 \\ 313-763-6225
}

\section{Extended Summary}

Observation and interaction are fundamental aspects of empirical scientific research. While individual requirements for observation and interaction vary across disciplines, there are important common elements. These "common elements" are the basis of the $\$ 15$ billion dollar laboratory automation industry. The United States presently leads the world in this industry, providing products for sample manipulation, sensing (e.g. microscopic and macroscopic imaging devices), data analysis (e.g. image processing, 3D modeling, exploratory data analysis), and humancomputer interfaces (e.g. computer graphics, visualization).

This paper outlines the preliminary design of a unique and innovative system for conducting experiments at a distance, the "Remote Scientist". The Remote Scientist extends laboratory automation capability to the microgravity environment. While the Remote Scientist conceptually encompasses a broad spectrum of elements and functionalities, the development approach taken is to:

- establish a baseline capability that is both flexible and versatile

- incrementally augment the baseline with additional functionalities over time.

The baseline capability that we have identified as having the most significant impact across multiple research disciplines is the ability to observe on-going experiments during exposure to the microgravity environment. This capability is achievable with technology available today and, additionally, will be able to take advantage of the rapid advances in the underlying technologies that are taking place in the commercial sector.

The Remote Scientist will support a wide range of experiments through customizable components for:

- data collection (observation and measurement)

- manipulation (automated and semi-automated experiment control)

- investigator interaction (communication between experiment and researcher independent of location)

By designing for multiple levels of automation, from fully automatic action through remote control and telemanipulation, investigator interaction can be incorporated into experiments at any desired level. The Remote Scientist is designed to offer a full range of digital imaging on both microscopic and macroscopic scales. The system will support spatial and spectral analysis for on-board extraction of important features, thus reducing bandwidth requirements for communications to ground-based investigators. Confocal imaging (optical sectioning), hyperspectral imaging, interferometry, laser scattering, and fluorescence can also be considered as value-added instrumentation within the proposed framework without significant redesign.

The system is designed to operate within the constraints of a microgravity environment while offering innovative functionality to enhance ground-based experiments as well. The Remote Scientist is initially focused on applications currently being pursued in microgravity such as tissue culture, protein crystal growth and zeolite crystal growth. The real-world requirements of these biomedical and materials applications provide important insight in establishing design criteria for the system. However, the longer-term applications are envisioned to be much broader.

\section{BENEFITS OF THE REMOTE SCIENTIST}

The Remote Scientist is expected to offer a number of benefits in the UF phase of the ISS:

- Improved mass efficiency. Infrastructure which provides observational capabilities for multiple experiments will reduce the need for each payload to develop and provide its own services.

- Improved crew efficiency. The observational capabilities of the Remote Scientist will enable ground-based scientists to have more direct insight into on-going experiments, thereby reducing crew actions to those that 
are critical. Automated and semi-automated manipulation capabilities will help to even crew workload by managing experiments during times of peak demand.

Microgravity investigators from multiple disciplines will benefit from well-conceived and technologically advanced observational capabilities of the kind outlined here. Additionally, the Remote Scientist will provide continuity in research programs from current missions through the fully operational ISS. This will be of particular value to those private sector industries currently being cultivated with regard to the opportunities afforded by microgravity (e.g. pharmaceutical, biomedical, and petrochemical research and manufacturing). Further, there is a new and developing microgravity supplier industry, exemplified by such organizations as ITA, Spacehab, and McDonnell Douglas; these organizations offer specialized laboratory research services which can be augmented and expanded by the Remote Scientist.

NASA will benefit from this capability through enhancement and further cultivation of the private sector industry utilization of microgravity and through the continued growth of the microgravity laboratory service supplier industry. NASA microgravity science programs will also benefit from the availability of the automation capabilities of the Remote Scientist. Finally, NASA will benefit from continued development of a broad U.S.-based user constituency for ISS.

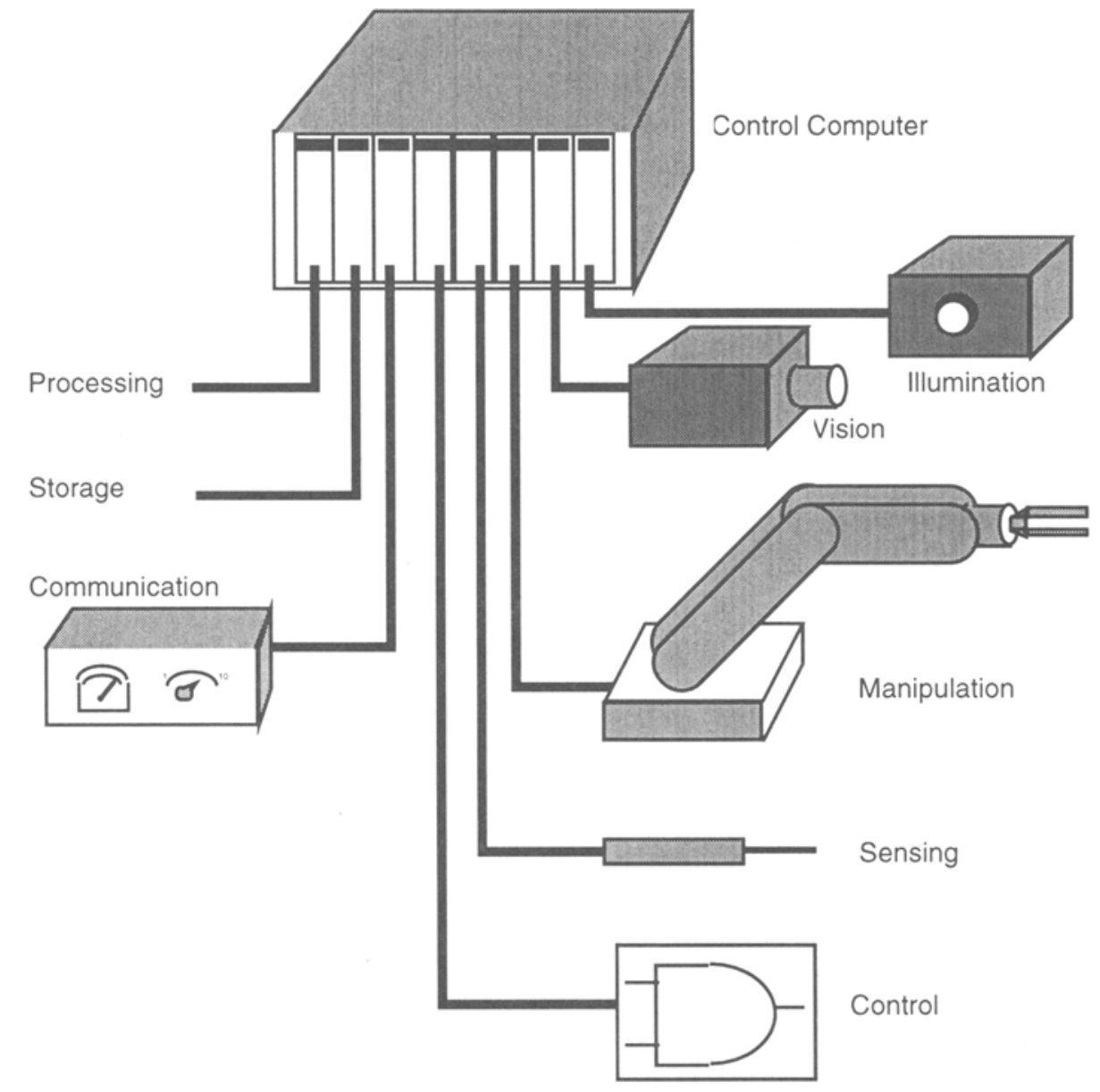

FIGURE 1. The Remote Scientist Block Diagram.

\section{SYSTEM OVERVIEW}

The Remote Scientist combines machine vision capabilities, motion control and digital and analog I/O in order to monitor and control experiments. For operation in microgravity, the system is designed to be small in both size and power consumption, to minimize power dissipation, and yet be customizable for a variety of experiments. The Remote Scientist will easily fit the form-factor constraints of 2 mid-deck locker equivalents and might be integrated as part of the "Rack Lite" concept. A preliminary block diagram for the Remote Scientist is shown in Figure 1. 
At the heart of the Remote Scientist is a control computer, the "brain" of the Remote Scientist. The control computer accepts 8 plug-in boards to handle:

- processing

- data storage

- communications

- illumination

- vision and other sensing

- manipulation

- device control

The components of the Remote Scientist are controlled through the use of LabView, an industry-standard laboratory information management system with extensions to support the specific requirements of the Remote Scientist. Off-the-shelf hardware components are integrated into the Remote Scientist through commercial plug-in boards to maximize the versatility and replicability of the system.

The Control Computer is based on the CompactPCI bus, the industrial version of the PCI bus popularized by desktop computers. The CompactPCI bus provides desktop-computer performance in a rugged enclosure. Flexibility and the ability to adapt to the unique interaction and manipulation requirements of each experiment is achieved through the use of Industry Packs (IP). The use of industry standard operating systems and LabView simplify the customization of experiment design and control.

The Remote Scientist offers an integrated solution to experiment automation. Like a real scientist, the Remote Scientist has the ability to see, to sense its environment, to take action based on its input and to communicate its findings to other scientists. In order to accommodate a wide variety of experiments, the Remote Scientist is modular; its size and capabilities are matched to the requirements of a particular experiment or series of tasks. For example, a simple Remote Scientist configuration may only require sensing and recording at fixed time intervals; a more complex task may require sophisticated imaging, image analysis, and control. A fully outfitted Remote Scientist in orbit will be able to work interactively with experimenters on the ground; ground-based systems will be able to interact with researchers at remote locations. 